\title{
Immediate and delayed impact of oral glucocorticoid therapy on risk of serious infection in older patients with rheumatoid arthritis: a nested case-control analysis
}

\author{
William G Dixon, ${ }^{1}$ Michal Abrahamowicz, ${ }^{2,3}$ Marie-Eve Beauchamp, ${ }^{3}$ David W Ray, ${ }^{4}$ \\ Sasha Bernatsky, ${ }^{5}$ Samy Suissa, ${ }^{6}$ Marie-Pierre Sylvestre ${ }^{7,8}$
}

- Additional supplementary materials are published online only. To view the files please visit the journal online (http://ard.bmj.com/ content/71/7.toc).

\section{${ }^{1}$ Arthritis Research UK}

Epidemiology Unit, Manchester Academic Health Science

Centre, University of Manchester, Manchester, UK 2Department of Epidemiology, Biostatistics and Occupational Health, McGill University,

Montreal, Canada

${ }^{3}$ Division of Clinical

Epidemiology, McGill University Health Center, Montreal, Canada ${ }^{4}$ Endocrine Sciences Research Group, Manchester Academic Health Science Centre, University of Manchester, Manchester, UK

${ }^{5}$ McGill University Health Centre, Department of Medicine, Divisions of Clinical Epidemiology and Rheumatology, Montreal, Canada ${ }^{6}$ Centre For Clinical Epidemiology, Lady Davis Institute - Jewish General Hospital, McGill University, Montreal, Canada ${ }^{7}$ Research Centre of the University of Montreal Hospital Centre, Montreal, Canada ${ }^{8}$ Department of Social and Preventive Medicine, Université de Montréal, Montréal, Canada

\section{Correspondence to} William Dixon, Arthritis Research UK Epidemiology Unit, Manchester Academic Health Science Centre, University of Manchester, Manchester, Stopford Building, Manchester M13 9PT, UK:

will.dixon@manchester.ac.uk

Received 28 November 2011 Accepted 1 December 2011 Published Online First: 12 January 2012

\section{UNIOCKID}

This paper is freely available online under the BMJ Journals unlocked scheme, see http:// ard.bmj.com/info/unlocked.dtl

\section{ABSTRACT}

Objectives To explore the relationship of serious infection risk with current and prior oral glucocorticoid (GC) therapy in elderly patients with rheumatoid arthritis (RA).

Methods A case-control analysis matched 1947 serious infection cases to five controls, selected from 16207 RA patients aged $\geq 65$ between 1985-2003 in Quebec, Canada. Adjusted odds ratios for infection associated with different GC patterns were estimated using conventional models and a weighted cumulative dose (WCD) model.

Results The WCD model predicted risks better than conventional models. Current and recent GC doses had highest impact on current risk. Doses taken up to 2.5 years ago were also associated with increased risk, albeit to a lesser extent. A current user of $5 \mathrm{mg}$ prednisolone had a $30 \%, 46 \%$ or $100 \%$ increased risk of serious infection when used continuously for the last 3 months, 6 months or 3 years, respectively, compared to a non-user. The risk associated with $5 \mathrm{mg}$ prednisolone taken for the last 3 years was similar to that associated with $30 \mathrm{mg}$ taken for the last month. Discontinuing a two-year course of $10 \mathrm{mg}$ prednisolone six months ago halved the risk compared to ongoing use.

Conclusions GC therapy is associated with infection risk in older patients with RA. The WCD model provided more accurate risk estimates than conventional models. Current and recent doses have greatest impact on infection risk, but the cumulative impact of doses taken in the last $2-3$ years still affects risk. Knowing how risk depends on pattern of GC use will contribute to an improved benefit/harm assessment.

Glucocorticoid therapy continues to be widely used as a treatment for patients with rheumatoid arthritis (RA). ${ }^{12}$ Although effective, ${ }^{34}$ there are concerns about infection and other safety outcomes including cardiovascular disease, diabetes, weight gain, cataracts and osteoporosis. ${ }^{5}$ Surprisingly, after over 60 years of experience, it is not clear how the risk of these outcomes depends on treatment regimes, what degree of risk is conferred, or what happens to risk on stopping therapy. We cannot accurately inform patients of the potential harms (as suggested in recent guidelines) ${ }^{6}$ if the risks are not accurately quantified.
Serious infection is one of the major causes of increased mortality in patients with RA.? Glucocorticoid therapy is a likely mediator as it impairs phagocyte function and suppresses cellmediated immunity. ${ }^{8}$ High-dose glucocorticoid therapy is associated with an increased risk of infection in other diseases, ${ }^{9}$ but the relationship with lower doses used in RA is less clear. ${ }^{10}$

Existing methods to quantify infection risk with glucocorticoid therapy have notable limitations. Randomised controlled trials in RA have small numbers of patients (only one $>250$ patients), ${ }^{11}$ short follow-up, and variable reporting of safety outcomes. ${ }^{10}$ Observational studies typically overcome all three limitations. Their 'real-life' setting, however, introduces analytical challenges. In addition to confounding, the lack of a prespecified treatment protocol means, particularly for glucocorticoid therapy, that treatment regimens vary between patients and change with time. Previous observational studies reporting glucocorticoid-associated infection risk have used exposure models such as 'current use', 'recent use', 'ever use' or 'total past dose'. ${ }^{12-17}$ These models ignore patterns of drug use with time; an important factor for glucocorticoid therapy in RA in which doses are continually increased, reduced or stopped. Furthermore, most studies select a priori one or, at most, few models for glucocorticoid exposure representation, and do not attempt to investigate if alternative models may fit their data better. Novel analytical methods exist to model a past history of drug use flexibly up to the time point when risk is being assessed. ${ }^{18}$ These methods represent exposure history by the weighted cumulative dose (WCD) of the drug of interest, with previous doses weighted according to their recency. ${ }^{19}$

The aim of this study was therefore to examine the association between the risk of serious infection and real-life patterns of glucocorticoid therapy in patients with RA. To this end, we performed a nested case-control analysis using data from a Canadian administrative database. To account for variation in the dosage, duration and timing of glucocorticoid therapy, and to explore how the risk depends on the treatment regimen, we employed the WCD model and compared the results with traditional approaches. 
Table 1 Baseline characteristics of cases and controls

\begin{tabular}{|c|c|c|}
\hline & Cases & Controls \\
\hline Number & 1947 & 9735 \\
\hline Age in years, median (IOR)* & $74(70-79)$ & $74(70-79)$ \\
\hline Sex $(\% \text { women })^{*}$ & 66.1 & 66.1 \\
\hline Follow-up in years, median (IOR)* & $3.0(1.2-5.8)$ & $3.0(1.2-5.8$ \\
\hline No of rheumatologist visits in the 6 months before index date, median (IOR) & $0(0-2)$ & $0(0-1)$ \\
\hline NSAID prescription within past 45 days (\%) & 52.5 & 46.7 \\
\hline \multicolumn{3}{|l|}{ DMARD prescription within past 45 days $(\%) \dagger$} \\
\hline Methotrexate & 30.1 & 27.5 \\
\hline Sulfasalazine & 1.8 & 2.1 \\
\hline Leflunomide & 0.6 & 0.3 \\
\hline Chloroquine/hydroxychloroquine & 20.9 & 21.6 \\
\hline Azathioprine & 2.8 & 1.5 \\
\hline Cyclophosphamide & 2.3 & 0.5 \\
\hline Anti-TNF therapy & 0.3 & 0.1 \\
\hline Gold & 4.8 & 5.5 \\
\hline Others (ciclosporin, mycophenolate mofetil, D-penicillamine) & 1.1 & 1.0 \\
\hline No of hospital admissions in year preceding index date, median (IOR) & $0(0-1)$ & $0(0-1)$ \\
\hline No of GP visits in year preceding index date, median (IOR) & $7(3-14)$ & $5(1-9)$ \\
\hline $\begin{array}{l}\text { No of hospital specialist visits (excluding rheumatologist) in year preceding } \\
\text { index date, median (IQR) }\end{array}$ & $8(3-16)$ & $5(2-10)$ \\
\hline \multicolumn{3}{|l|}{ Co-morbidity (from ICD-9 or drug codes) (\%) } \\
\hline Chronic respiratory disease (COPD/asthma/LD) & 26.5 & 15.7 \\
\hline Osteoporosis & 24.8 & 17.5 \\
\hline Chronic renal disease & 0.4 & 0.1 \\
\hline Cancer & 4.3 & 1.7 \\
\hline Diabetes & 8.4 & 6.0 \\
\hline $\mathrm{PPI} / \mathrm{H}_{2}$ blocker prescription within past 45 days (\%) & 36.6 & 24.1 \\
\hline
\end{tabular}

*Variables used for matching.
tPatients could be on more than one DMARD and contribute to more than one category.
COPD, chronic obstructive pulmonary disease; DMARD, disease-modifying antirheumatic drug; GP, general practitioner; ILD,
interstitial lung disease; NSAID, non-steroidal
anti-inflammatory drug; PPI, proton-pump inhibitor; TNF, tumour necrosis factor.

\section{PATIENTS AND METHODS}

\section{Study base}

Patients with RA were identified from the administrative databases of the Régie de l'assurance maladie du Québec and the Maintenance et Exploitation des Données pour l'Étude de la Clientèle Hospitalière, in Quebec, Canada. ${ }^{16}$ Briefly, linked data are available on demographics, medical services including diagnostic codes for billed services and hospital admission data. For patients aged 65 years and older, data were available on date, duration and dose of each prescription dispensed.

\section{Study population}

The sampling frame consisted of all patients with RA aged 65 years and older who, between 1 January 1985 and 31 December 2003, were dispensed at least one traditional or biological diseasemodifying antirheumatic drug (DMARD). RA was identified on the basis of at least one physician billing code (International Classification of Disease, version 9 (ICD-9) code 714). Cohort entry was defined as the date of first DMARD prescription following an RA diagnosis. All subjects were followed from cohort entry date to the earliest of serious infection, death or the end of the study period (31 March 2004). Subjects were required to have more than 3 months of eligibility in the health insurance plan before cohort entry.

\section{Definition of cases and controls}

Serious infection cases were identified as the first occurrence of a primary hospital discharge diagnosis of infection (see supplementary file for ICD codes, available online only), which defined the index date. For each case of serious infection, we identified a risk set of patients with RA who joined the cohort on the same date following an RA code and DMARD use, had the same sex and similar age, had not developed a serious infection by the corresponding index date, but who were still under follow-up. For each case, from the respective risk set we randomly selected five controls. Controls could later be included as cases, and the same patient could be selected as a control for different cases.

\section{Exposure}

All drugs dispensed before the index date were identified from the prescription database. Systemic glucocorticoid therapy (oral, intravenous or intramuscular injection) was identified using the drug product database of Health Canada. ${ }^{20}$ Oral glucocorticoid dosage was averaged across each prescription period (derived directly from the prescription database) and converted to daily prednisolone equivalent dosage (PEQ). Injectable (intramuscular or intravenous) glucocorticoid therapy was adjusted for in the analysis using the number of prescriptions of injectable glucocorticoids in each of the three time periods: 0-6 months; 7-12 months and $1-5$ years.

\section{Confounders}

Disease severity, other immunosuppressants and co-morbidity were potential confounders. In the absence of direct measures of disease severity, surrogate markers were used (number of rheumatologist visits in the preceding year, current non-steroidal antiinflammatory drug (NSAID) use). ${ }^{21}$ All analyses were adjusted for concomitant DMARD exposure, defined as a DMARD prescription in the 45 days before the index date. Comorbidity was identified from diagnoses before the index date (chronic respiratory conditions, osteoporosis, cancer, diabetes and chronic renal 
Table 2 Relationship between serious infection risk and oral glucocorticoid exposure for the 10 conventional models and the best fitting cumulative weighted dose model

\begin{tabular}{|c|c|c|c|c|c|c|}
\hline Model & $\begin{array}{l}\text { Among cases } \\
(\% \text { or mean })^{*}\end{array}$ & $\begin{array}{l}\text { Among controls } \\
(\% \text { or mean })^{*}\end{array}$ & OR $(95 \% \mathrm{Cl}) \dagger$ & $\begin{array}{l}\text { OR for } 5 \text { mg increase } \\
(95 \% \mathrm{Cl})\end{array}$ & AIC $¥$ & $\begin{array}{l}\text { AIC-AIC of the WCD } \\
\text { model }\end{array}$ \\
\hline \multicolumn{7}{|l|}{ Binary conventional models } \\
\hline (1) Current use & $42.1 \%$ & $24.7 \%$ & 1.84 (1.64 to 2.06$)$ & - & 6175.0 & 115.2 \\
\hline (2) Any use past 30 days & $52.9 \%$ & $31.1 \%$ & $2.08(1.86$ to 2.33$)$ & - & 6122.1 & 62.3 \\
\hline (3) Any use past 90 days & $58.6 \%$ & $34.7 \%$ & 2.26 (2.02 to 2.54$)$ & - & 6083.6 & 23.8 \\
\hline (4) Ever use & $74.4 \%$ & $58.5 \%$ & $1.72(1.53$ to 1.94$)$ & - & 6201.5 & 141.7 \\
\hline \multicolumn{7}{|l|}{ Dose-specific conventional models } \\
\hline (5) Current dose $\mathrm{e}^{\mathrm{a}}$ & 11.0 mg PEQ & $7.8 \mathrm{mg}$ PEO & 1.04 (1.04 to 1.05$)$ & 1.24 (1.19 to 1.30$)$ & 6171.3 & 111.4 \\
\hline (6) Average dose in past 30 days $^{b}$ & $8.3 \mathrm{mg}$ PEO & $6.2 \mathrm{mg}$ PEO & $1.07(1.06$ to 1.08$)$ & 1.41 (1.33 to 1.49$)$ & 6124.0 & 64.2 \\
\hline (7) Average dose in past 90 days $^{b}$ & $7.1 \mathrm{mg}$ PEQ & $5.4 \mathrm{mg}$ PEO & 1.09 (1.08 to 1.11$)$ & 1.56 (1.46 to 1.67$)$ & 6094.8 & 34.9 \\
\hline (8) Average dose since study entry ${ }^{b}$ & 5.4 mg PEQ & $3.7 \mathrm{mg}$ PEO & $1.08(1.06$ to 1.09$)$ & $1.45(1.35$ to 1.56$)$ & 6166.6 & 106.8 \\
\hline (9) Peak dose in past 30 days $^{c}$ & 13.6 mg PEQ & $9.3 \mathrm{mg}$ PEO & $1.03(1.02$ to 1.03$)$ & $1.14(1.10$ to 1.17$)$ & 6187.3 & 127.5 \\
\hline (10) Peak dose in past 90 days $^{c}$ & 15.2 mg PEQ & 10.9 mg PEQ & $1.02(1.01$ to 1.02$)$ & $1.10(1.07$ to 1.12$)$ & 6200.3 & 140.5 \\
\hline \multicolumn{7}{|c|}{ Flexible model incorporating weighting by recency of treatment } \\
\hline $\begin{array}{l}\text { (11) Final WCD (3-year with } 3 \text { degrees } \\
\text { of freedom) }\end{array}$ & $\S$ & $\S$ & $\S$ & $\S$ & 6059.8 & 0 (minimum AIC) \\
\hline
\end{tabular}

OR, adjusted for all a priori confounders.

*For models representing exposure by binary indicator variables (1-4), the value provided is the proportion of cases/controls with the defined glucocorticoid exposure. For models with continuous exposure (5-10), the mean value of the exposure variable among glucocorticoid therapy users for cases/controls is given (see ${ }^{\mathrm{a}-\mathrm{c}}$ below).

†For dose-specific models (5-10), OR represents risk per $1 \mathrm{mg}$ PEQ increase.

$\ddagger$ A lower AIC value indicates a better fit to data.

$\S B$ cause the (non-parametric) WCD model estimates exposure effect using flexible spline functions, the estimated effect cannot be summarised by a single parameter. See figure 1 for the estimated weight function and table 3 for the WCD model estimates of adjusted OR associated with selected exposure patterns.

aMean current dose $=$ mean dose in current users.

${ }^{b}$ Average dose in past 30/90 days/since study entry=average dose over the past 30/90 days/since study entry in subjects who used glucocorticoid therapy for at least 1 day in past $30 / 90$ days since study entry.

'Mean peak dose in past 30/90 days =mean peak dose in past 30/90 days in subjects who used glucocorticoid therapy for at least 1 day in past $30 / 90$ days

AIC, Akaike information criterion; PEQ, prednisolone equivalent; WCD, weighted cumulative dose.

disease). These comorbidities were also identified from medication typically associated with their treatment. Diabetes was included as a confounder only if the onset of diabetes preceded the first glucocorticoid prescription, thereby avoiding diabetes being on a causal pathway from glucocorticoid use to infection. All models also adjusted for age, number of hospital admissions, number of general practitioner (GP) and hospital specialist visits in the preceding year and concomitant use of gastric acid-suppressive drugs. ${ }^{22}$

\section{Statistical analyses}

Primary analyses assessed the impact of glucocorticoid exposure on serious infection risk. We employed multivariable conditional logistic regression to contrast the exposure patterns between cases and their matched controls. Because of the uncertainty about the mechanisms linking glucocorticoid exposure and the risk of serious infection, we estimated several multivariable models, each representing glucocorticoid exposure differently. All available cases were included rather than performing a formal sample size calculation to determine study size.

\section{Conventional models}

The first 10 models used conventional exposure metrics often employed in pharmacoepidemiology. Models 1-4 disregarded information on glucocorticoid dose and represented exposure by binary indicator variables of: (1) current use on the index date; or any use in: (2) the previous 30 days; (3) 90 days; (4) or any time in the past (indicator of ever exposed). Model 5 used a quantitative measure of current daily oral dose at the index date. Models 6-8 defined the exposure metric as average past oral dose taken over a relevant time window, corresponding to previous (6) 30 days or (7) 90 days, or (8) the entire time since the subject's entry into the cohort. Models 9-10 used the peak oral dose over (9) the previous 30 days or (10) 90 days.

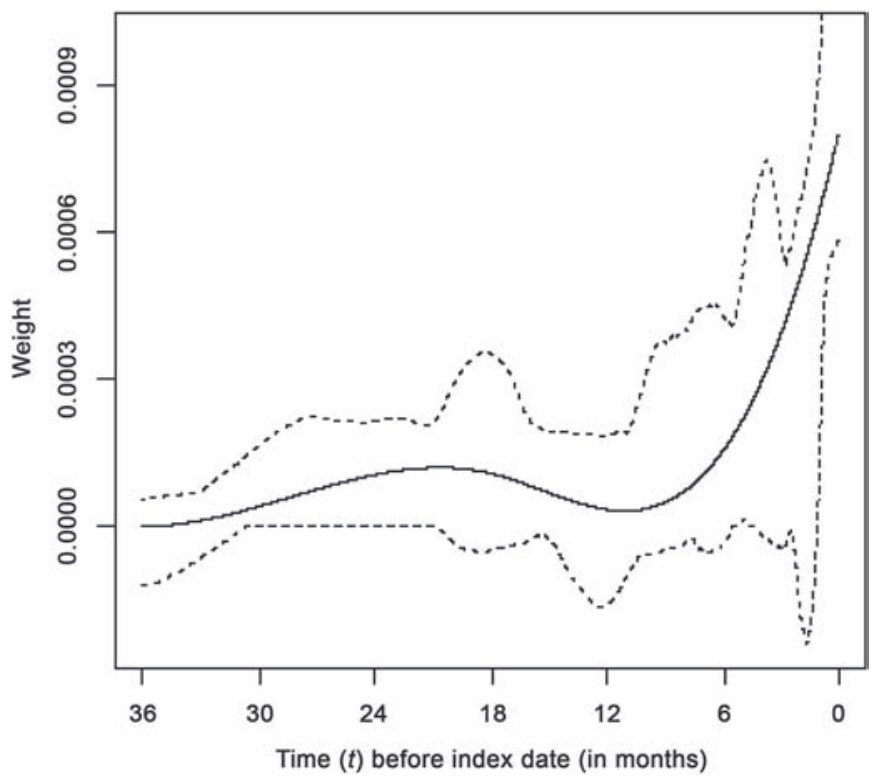

Figure 1 Estimated weight function (solid curve) and pointwise $95 \%$ bootstrap $\mathrm{Cl}$ (dotted curves) for the final weighted cumulative dose model of the association between previous oral glucocorticoid exposure and serious infection. The final WCD model used 3 degrees of freedom to model the weight function (see Supplementary Online Materials). Relative risks associated with specific exposure patterns are derived from the weighted sum of past doses, with weights shown on the $y$-axis. Accordingly, the total impact of continuing exposure (in terms of $\log \mathrm{OR}$ ) during a given time period corresponds to the area under the weight curve (over the corresponding time interval).

\section{Flexible weighted cumulative dose model}

Finally, in the WCD model, ${ }^{19}$ glucocorticoid exposure was represented as the weighted sum of past oral doses, with weights estimated so as to reflect the relative importance of 
Table 3 Adjusted OR (with 95\% bootstrap Cl) for the association between various clinical patterns of glucocorticoid therapy during the past 3 years and risk of current serious infection

\begin{tabular}{|c|c|c|}
\hline Pattern of use & Reference & Adjusted OR $(95 \% \mathrm{CI})^{*}$ \\
\hline Current user, $5 \mathrm{mg}$, for past 7 days & Non-user & $1.03(1.02$ to 1.11$)$ \\
\hline Current user, $5 \mathrm{mg}$, for past 28 days & Non-user & $1.11(1.08$ to 1.26$)$ \\
\hline Current user, $5 \mathrm{mg}$, for past 3 months & Non-user & $1.30(1.21$ to 1.45$)$ \\
\hline Current user, $5 \mathrm{mg}$, for past 6 months & Non-user & $1.46(1.31$ to 1.65$)$ \\
\hline Current user, $5 \mathrm{mg}$, for past 1 year & Non-user & 1.55 (1.41 to 1.88$)$ \\
\hline Current user, $5 \mathrm{mg}$, for past 3 years & Non-user & $2.00(1.69$ to 2.26$)$ \\
\hline Past user, $5 \mathrm{mg}$, for 6 months, stopped 6 months ago & Non-user & $1.06(0.98$ to 1.27$)$ \\
\hline Current user, $5 \mathrm{mg}$, for past 6 months & Past user, $5 \mathrm{mg}$, for 6 months, stopped 6 months ago & $1.38(1.07$ to 1.61$)$ \\
\hline Current user, 30 mg, for past 7 days & Non-user & $1.18(1.13$ to 1.86$)$ \\
\hline Current user, $30 \mathrm{mg}$, for past 28 days & Non-user & 1.84 (1.58 to 4.00$)$ \\
\hline Current user, $30 \mathrm{mg}$, for past 3 months & Non-user & 4.82 (3.12 to 9.29$)$ \\
\hline Current user, $30 \mathrm{mg}$, for past 6 months & Non-user & 9.81 (5.13 to 19.92$)$ \\
\hline Current user, $30 \mathrm{mg}$, for past 30 days & Past user, $30 \mathrm{mg}$, for 30 days, stopped 14 days ago & 1.07 (1.04 to 2.87$)$ \\
\hline Current user, $30 \mathrm{mg}$, for past 30 days & Past user, $30 \mathrm{mg}$, for 30 days, stopped 28 days ago & $1.13(1.07$ to 4.30$)$ \\
\hline Current user, $30 \mathrm{mg}$, for past 30 days & Past user, $30 \mathrm{mg}$, for 30 days, stopped 3 months ago & $1.40(1.20$ to 3.66$)$ \\
\hline Current user, $30 \mathrm{mg}$, for past 14 days & Past user, 30 mg, for 14 days, stopped 28 days ago & 1.06 (1.03 to 2.79$)$ \\
\hline Current user, $10 \mathrm{mg}$, for past 2 years & Past user, $10 \mathrm{mg}$, for 2 years, stopped 2 weeks ago & $1.10(1.06$ to 1.41$)$ \\
\hline Current user, $10 \mathrm{mg}$, for past 2 years & Past user, $10 \mathrm{mg}$, for 2 years, stopped 1 month ago & $1.20(1.12$ to 1.61$)$ \\
\hline Current user, $10 \mathrm{mg}$, for past 2 years & Past user, $10 \mathrm{mg}$, for 2 years, stopped 3 months ago & 1.55 (1.33 to 2.03$)$ \\
\hline Current user, $10 \mathrm{mg}$, for past 2 years & Past user, 10 mg, for 2 years, stopped 6 months ago & 1.87 (1.32 to 2.63$)$ \\
\hline
\end{tabular}

${ }^{*}$ Adjusted OR represents the relative risk of infection for the pattern of use in column one compared with the reference pattern of use in column two.

doses taken at different times. The weights assigned to past doses were estimated using a flexible cubic spline technique that avoided a priori assumptions regarding the shape of the weight function. ${ }^{23}$

Because of the uncertainty regarding the time window over which past glucocorticoid exposure may affect the current risk of serious infection, six alternative WCD models with exposure time windows corresponding to previous 1 or 3 months, or 1 , 2,3 or 4 years were fitted. Procedure and criteria used to select the final WCD model are described in detail in the supplementary materials, available online only. Models' goodness of fit was compared based on the minimum Akaike information criterion (AIC). ${ }^{24}$ For benchmarking, any AIC difference bigger than 10 is considered very important. ${ }^{25}$

\section{RESULTS}

Among 16207 patients with RA aged 65 years or older, 1947 developed a first serious infection after an average of 3.8 years follow-up. These were matched to 9735 controls, each case successfully matched to five controls. Cases had more severe disease as evidenced by a higher number of rheumatologist and GP visits in the preceding year and more NSAID use (table 1). Cases also had higher levels of comorbidity. There were no missing data.

The relationships between serious infection and glucocorticoid exposure, estimated through the 10 conventional models, are described in table 2 . All models indicated statistically significant associations between glucocorticoid exposure and serious infection $(\mathrm{p}<0.0001$, data not shown). However, the goodness of fit, quantified by AIC, of different models varied considerably depending on the way glucocorticoid exposure was represented, the time window over which it was considered, and whether the glucocorticoid doses were taken into account. Among the conventional models, the best fit (lowest AIC) was obtained with any use in the past 90 days (model 3), followed by average dose in the past 90 days (model 7). Average dose, or any use, in the past 30 days (models 6 and 2 ) provided the next best fit. All other conventional models including current use, ever use and average past dose (models 1, 4 and 8) provided a notably worse fit (table 2).
Among the alternative WCD models, those estimating weighted cumulative exposure over the past 1-4 years fitted the data much better than those assuming shorter windows of aetiologically relevant exposure (data not shown). The best-fitting final WCD model corresponded to the 3-year window model. The final WCD model predicted serious infections much better than any of the conventional models, whose AIC were more than 20-140 units higher (table 2), recalling that a 10 unit difference is considered very important. ${ }^{25}$ As explained in section 3 of the supplementary materials (available online only), such differences may be interpreted as the evidence that the WCD model provides statistically very significant improvements in the prediction of outcomes, compared with all conventional models (all $\mathrm{p}$ values $<0.0001)$.

The estimated weight function for the final best-fitting flexible WCD model is shown in figure 1. The horizontal axis shows time $(t)$ before the index date and the vertical axis shows the corresponding estimated weights, reflecting the relative strength of the impact of glucocorticoid doses taken $t$ months ago on the current risk of infection. As expected, the current and very recent doses (near $t=0$ ) have the highest impact on current risk, and the effect of past doses decreases sharply with increasing time since exposure. Still, even doses taken approximately half a year ago seem to have some impact on the current risks. Interestingly, the weight function suggests that, in addition to the effect of glucocorticoid exposure in the past 6 months, doses taken up to 2.5 years ago are also associated with increased current risk. These 'remote' doses have relatively low weights but, if taken for a long time, can lead to an important cumulative effect.

In sensitivity analyses, we re-estimated the WCD models for two separate subsamples, each of which was restricted to the case-control risk sets corresponding to infections that occurred before or after 1 January 1996 (565 and 1382 cases, respectively). The estimated weight functions obtained for both calendar year-stratified analyses were quite similar to the estimate for the entire sample, shown in figure 1 (data not shown). This indicated that our estimates are not materially affected by potential changes in glucocorticoid prescribing that may have occurred during the study period (1985-2004). Furthermore, to verify the 
shape of the weight function, we estimated models in which glucocorticoid exposure was represented by a series of seven separate variables, each corresponding to a specific (mutually exclusive) time interval (past 3 months, 3-6 months ago, 6-12 months ago, ..., 30-36 months ago). Glucocorticoid exposure was modelled for each of these intervals using binary indicators of 'any use' (similar to models $2-3$ in table 2 ) or 'average dose' (similar to models 6-7). Reassuringly, consistent with our final WCD model, the strongest association was found for doses taken in the most recent 3 months, a weaker association for months 3-6 and 6-12, no association for months 12-18, a return of a weak positive association in months 18-24 and 24-30, and a decay to no association at months 30-36 (further details in the supplementary material, available online only).

Table 3 illustrates the implications of the WCD model for assessing relative risks associated with different patterns of past glucocorticoid exposure. The left-most column describes selected, clinically plausible patterns of current and past glucocorticoid doses. The second column describes the comparator ('reference') pattern, either a 'non-user', ie, a subject who did not use glucocorticoids throughout the past 3 years or a subject who had a similar dose and duration of glucocorticoid treatment in the more distant past. The right column shows the corresponding adjusted $\mathrm{OR}$, estimated through the final WCD model that compares the risks between the two patterns in the same row. For example, a subject who was taking a daily dose of $5 \mathrm{mg}$ PEQ for the past 6 months is estimated to have a $46 \%$ higher risk (OR 1.46) than a non-user (4th row, table 3). Different categories in table 3 are not mutually exclusive. For example, the OR for $5 \mathrm{mg}$ PEQ taken for the past 6 months is a combination of the risk associated with $5 \mathrm{mg}$ PEQ taken for the past 3 months (reported in the line above, OR 1.30) plus the risk from $5 \mathrm{mg}$ $\mathrm{PEQ}$ taken 3-6 months ago. Overall, table 3 demonstrates that the risks vary substantially with dosage, duration and timing of the glucocorticoid treatment, and that the timing of discontinuation has a notable impact on infection risk.

\section{DISCUSSION}

This study confirms that glucocorticoid therapy is associated with serious infection in older patients with RA. We have replicated previous reports of an increased risk using conventional models of exposure. For example, we have shown in model 1 an OR for current exposure of 1.84 (95\% CI 1.64 to 2.06), which compares with previous estimates using the same analysis model of between 1.9 and 2.1. ${ }^{13-15}$ Similarly, our OR for recent exposure $(2.26,95 \%$ CI 2.02 to 2.54 , model 3 ) is similar to a previous estimate of 2.56 (95\% CI 2.29 to 2.85$),{ }^{16}$ and for ever exposed our estimate of 1.72 (95\% CI 1.53 to 1.94$)$ from model 4 overlaps with a published estimate of 2.2 (95\% CI 1.5 to 3.4). ${ }^{12}$ Furthermore, our dose-specific estimates are also similar to those published analyses that accounted for glucocorticoid doses. In our study, $5 \mathrm{mg}$ PEQ was associated with an OR of 1.4-1.6 (models 6 and 7 in table 2), compared with previous estimates of 1.3-1.5 for 1-5 mg PEQ and 1.5-2.1 for 5-10 mg PEQ. ${ }^{14}$ 152627 More importantly, however, our knowledge of the relationship between dynamic glucocorticoid therapy and infection risk has been extended by applying novel methodology. Indeed, our results clearly demonstrate the importance of the cumulative effects of the past use of glucocorticoids on serious infection risk, and accounting for differential impact of glucocorticoid doses taken at different times in the past.

Conventional drug exposure models rely on rather improbable assumptions about the relative importance of previous therapy. 'Current exposure' assumes any previous treatment has no effect on infection risk. A 'recent exposure' model considering use in the past 30 days assumes treatment received 29 days ago is important but 31 days ago carries no risk. In 'ever exposed' models, $10 \mathrm{mg}$ PEQ taken several years ago carries the same risk as $10 \mathrm{mg}$ taken now. Because previous analyses have tended to select only one of these models, clinicians are left uncertain about the temporal relationship between glucocorticoid use and infection. In contrast, our flexible WCD model ${ }^{19}$ accounts directly for the variation in glucocorticoid timing by assigning differential weights to doses taken at different time intervals in the past.

The WCD model provided a far better fit to our data than any of the conventional models and, thus, provided strong evidence that the risk of infection cumulated over past glucocorticoid use but also varied with the dose and recency of treatment. We have shown that cumulative effects affect the risk of infection even 2-3 years later and accounting for such long-term effects improves risk prediction. For example, whereas $5 \mathrm{mg}$ PEQ carries a $11 \%, 30 \%$ or $55 \%$ increased risk of infection compared with non-users if taken for 1, 3 or 12 months, respectively, the same dose taken for 3 years would lead to a $100 \%$ risk increase (table 3 ). This additional increase in risk from $55 \%$ to $100 \%$, when extending continuing glucocorticoid exposure from 1 to 3 years, results from the cumulative effect of long-term exposure over two additional years, even though the weights assigned to doses taken $1-3$ years ago are significantly lower than the weights assigned to more recent doses (figure 1). This is important as many physicians consider $5 \mathrm{mg} \mathrm{PEQ}$ a 'physiological dose' that may not have important harms. We have also been able to explore the impact of glucocorticoid discontinuation. For example, we have shown that $5 \mathrm{mg} \mathrm{PEQ}$ is associated with an OR of 1.46 compared with a non-user if used continuously for the past 6 months, but that this risk falls to only 1.06 if the same exposure occurred between 12 and 6 months ago, ie, was discontinued 6 months ago (table 3 ).

Knowledge of time-varying risk can contribute more fully to the benefit/harm balance of glucocorticoid use in clinical practice, for example before elective procedures. Continuing a 1-month course of $30 \mathrm{mg}$ prednisolone up to the date of surgery may be associated with up to a $13-40 \%$ higher risk than stopping 1-3 months earlier (table 3). Equally important, a shorter 2 -week course of $30 \mathrm{mg}$ prednisolone carries little additional risk if taken immediately before the date of interest, compared with a course of the same duration that is discontinued 1 month earlier.

Having defined the shape of the estimated weight function in figure 1, we should consider possible explanations for the pattern of the relationship between infection risk and current and past doses, be they causal or otherwise. As expected, current and recent glucocorticoid therapy has the strongest association with current risk. Glucocorticoids act on both the innate and adaptive immune system. Impaired innate immunity would be expected to have an immediate impact, but may be predicted to recover following glucocorticoid discontinuation. Our data show that 'remote' therapy has a lesser, but still important, impact. The effects on adaptive immunity, which include T-lymphocyte apoptosis, may be long-standing and include failure to generate pathogen-specific adaptive immune responses. ${ }^{28}$ Furthermore, trophic effects of glucocorticoids on connective tissue may impair barrier function and result in long-term changes in host vulnerability to infection, for example skin atrophy. The finding of a non-negligible, statistically significant effect of glucocorticoid use between 1 and 3 years ago on the current risk of serious infection may reflect an integrated effect of multiple glucocorticoid-regulated phenomena, each with a 
different recovery interval following discontinuation of therapy, as discussed above. A further and indirect mechanism may be the effect of glucocorticoids on the severity of, or host response to, infection, perhaps reflecting the consequences of prolonged adrenal suppression. Finally, glucocorticoid therapy may act as a marker for ongoing disease activity and/or disability. Patients who used glucocorticoid therapy 2 years ago may be more likely to have high current disability, which is a known risk factor for serious infection. ${ }^{29}$ We were unable to test the reasons for the pattern of association within this study, but future research may explore the above conjectures.

This observational study has some limitations. We did not have measures of adherence, and so our estimates derived from dispensing data may underestimate the true risk, even if they are unlikely to affect the comparisons between alternative models. Confounding is an important consideration. We have adjusted for concomitant DMARD therapies and co-morbidities that might be associated with both glucocorticoid exposure and infection. No direct measures of RA disease activity exist within the administrative database so, similar to other studies, we adjusted for surrogate measures including the number of rheumatologist visits and concomitant NSAID use. ${ }^{1630}$ While residual confounding by disease activity will remain, we have previously shown that it cannot fully explain the observed relationship between glucocorticoid therapy and infection risk. ${ }^{30}$ Importantly, it is unlikely to confound the comparisons of risks associated with different patterns of past glucocorticoid use and of goodness of fit of the different exposure models. ${ }^{1831}$

Comprehensive prescription data were available only for patients aged 65 years or older. While restriction to this age group may limit generalisability, we would not anticipate the shape of the relationship between glucocorticoid therapy and infection risk differing significantly in other age groups of patients with RA. Furthermore, replication of previous findings using conventional models is reassuring.

\section{CONCLUSION}

We have shown that a WCD model applied to glucocorticoid use in older patients with RA provides a far superior fit than all conventional models. The results confirm that glucocorticoid therapy is associated with an increased risk of infection when the risk is dependent on the recency of treatment. Contrary to the assumptions of many conventional models, doses 2 years ago still have an influence on infection risk.

Contributors WGD and MA conceived and designed the study; SS and SB acquired the data; MEB, MA and MPS analysed the data; WGD, MA and MEB interpreted the data and drafted the article; DWR, SS, SB and MPS contributed to interpretation of the data and critical revision of the manuscript for important intellectual content. All researchers had access to all the data in the study. WGD is the guarantor.

Funding The database acquisition was funded by a grant to SS from the Canadian Institutes of Health Research (CIHR) and the Canadian Foundation for Innovation. The analyses were supported by the CIHR grant MOP-81275 (PI: MA). WGD was supported by an MRC Clinician Scientist Fellowship (G0902272)

Competing interests None.

Ethical approval The McGill University Institutional Review Board approved the study. Provenance and peer review Not commissioned; externally peer reviewed.

\section{REFERENCES}

1. Caplan L, Wolfe F, Russell AS, et al. Corticosteroid use in rheumatoid arthritis: prevalence, predictors, correlates, and outcomes. J Rheumatol 2007;34:696-705.

2. Thiele K, Buttgereit F, Huscher D, et al. Current use of glucocorticoids in patients with rheumatoid arthritis in Germany. Arthritis Rheum 2005:53:740-7.
3. Gøtzsche PC, Johansen HK. Meta-analysis of short-term low dose prednisolone versus placebo and non-steroidal anti-inflammatory drugs in rheumatoid arthritis. BMJ 1998;316:811-18.

4. Kirwan JR, Bijlsma JW, Boers M, et al. Effects of glucocorticoids on radiological progression in rheumatoid arthritis. Cochrane Database Syst Rev 2007;1:CD006356.

5. Hoes JN, Jacobs JW, Buttgereit F, et al. Current view of glucocorticoid co-therapy with DMARDs in rheumatoid arthritis. Nat Rev Rheumatol 2010;6:693-702.

6. Hoes JN, Jacobs JW, Boers M, et al. EULAR evidence-based recommendations on the management of systemic glucocorticoid therapy in rheumatic diseases. Ann Rheum Dis 2007;66:1560-7.

7. Thomas E, Symmons DP, Brewster DH, et al. National study of cause-specific mortality in rheumatoid arthritis, juvenile chronic arthritis, and other rheumatic conditions: a 20 year followup study. J Rheumatol 2003;30:958-65.

8. Cutolo M, Seriolo B, Pizzorni C, et al. Use of glucocorticoids and risk of infections. Autoimmun Rev 2008;8:153-5.

9. Stuck AE, Minder CE, Frey FJ. Risk of infectious complications in patients taking glucocorticosteroids. Rev Infect Dis 1989;11:954-63.

10. Dixon WG, Suissa S, Hudson M. The association between systemic glucocorticoid therapy and the risk of infection in patients with rheumatoid arthritis: systematic review and meta-analyses. Arthritis Res Ther 2011;13:R139.

11. Choy EH, Smith CM, Farewell V, et al.; CARDERA (Combination Anti-Rheumatic Drugs in Early Rheumatoid Arhritis) Trial Group. Factorial randomised controlled trial of glucocorticoids and combination disease modifying drugs in early rheumatoid arthritis. Ann Rheum Dis 2008;67:656-63.

12. Franklin $\mathbf{J}$, Lunt $\mathrm{M}$, Bunn $\mathrm{D}$, et al. Risk and predictors of infection leading to hospitalisation in a large primary-care-derived cohort of patients with inflammatory polyarthritis. Ann Rheum Dis 2007;66:308-12.

13. Lacaille D, Guh DP, Abrahamowicz M, et al. Use of nonbiologic disease-modifying antirheumatic drugs and risk of infection in patients with rheumatoid arthritis. Arthritis Rheum 2008;59:1074-81.

14. Smitten AL, Choi HK, Hochberg MC, et al. The risk of hospitalized infection in patients with rheumatoid arthritis. J Rheumatol 2008:35:387-93.

15. Schneeweiss S, Setoguchi S, Weinblatt ME, et al. Anti-tumor necrosis factor alpha therapy and the risk of serious bacterial infections in elderly patients with rheumatoid arthritis. Arthritis Rheum 2007;56:1754-64.

16. Bernatsky S, Hudson M, Suissa S. Anti-rheumatic drug use and risk of serious infections in rheumatoid arthritis. Rheumatology (Oxford) 2007;46:1157-60.

17. Saag KG, Koehnke R, Caldwell JR, et al. Low dose long-term corticosteroid therapy in rheumatoid arthritis: an analysis of serious adverse events. Am J Med 1994;96:115-23.

18. Abrahamowicz M, Bartlett G, Tamblyn R, et al. Modeling cumulative dose and exposure duration provided insights regarding the associations between benzodiazepines and injuries. J Clin Epidemiol 2006;59:393-403.

19. Sylvestre MP, Abrahamowicz M. Flexible modeling of the cumulative effects of time-dependent exposures on the hazard. Stat Med 2009;28:3437-53.

20. Health Canada. Drug Product Database Online Query: http://webprod.hc-sc. gc.ca/dpd-bdpp/index-eng.jsp. 2010 [cited 2010 23/4/2010]; Available from: http:// webprod.hc-sc.gc.ca/dpd-bdpp/index-eng.jsp (accessed 23 April 2010)

21. Ting G, Schneeweiss S, Katz JN, et al. Performance of a rheumatoid arthritis recordsbased index of severity. J Rheumatol 2005;32:1679-87.

22. Laheij RJ, Sturkenboom MC, Hassing RJ, et al. Risk of community-acquired pneumonia and use of gastric acid-suppressive drugs. JAMA 2004;292:1955-60.

23. Abrahamowicz M, MacKenzie TA. Joint estimation of time-dependent and nonlinear effects of continuous covariates on survival. Stat Med 2007;26:392-408.

24. Akaike H. A new look at the statistical model identification. IEEE Trans Automat Contr 1974;19:716-23

25. Quantin C, Abrahamowicz M, Moreau T, et al. Variation over time of the effects of prognostic factors in a population-based study of colon cancer: comparison of statistical models. Am J Epidemiol 1999;150:1188-200.

26. Curtis JR, Patkar N, Xie A, et al. Risk of serious bacterial infections among rheumatoid arthritis patients exposed to tumor necrosis factor alpha antagonists. Arthritis Rheum 2007:56:1125-33.

27. Wolfe F, Caplan L, Michaud K. Treatment for rheumatoid arthritis and the risk of hospitalization for pneumonia: associations with prednisone, disease-modifying antirheumatic drugs, and anti-tumor necrosis factor therapy. Arthritis Rheum 2006;54:628-34.

28. McMaster A, Ray DW. Drug insight: selective agonists and antagonists of the glucocorticoid receptor. Nat Clin Pract Endocrinol Metab 2008;4:91-101.

29. Doran MF, Crowson CS, Pond GR, et al. Predictors of infection in rheumatoid arthritis. Arthritis Rheum 2002;46:2294-300.

30. Dixon WG, Kezouh A, Bernatsky S, et al. The influence of systemic glucocorticoid therapy upon the risk of non-serious infection in older patients with rheumatoid arthritis: a nested case-control study. Ann Rheum Dis 2011;70:956-60.

31. Abrahamowicz M, Beauchamp ME, Sylvestre MP. Comparison of alternative models for linking drug exposure with adverse effects. Stat Med. Published Online First: 17 November 2011. doi:10.1002/sim.4343. 